\title{
Supporting Information: Following Metal-to-Ligand Charge-Transfer Dynamics with Ligand and Spin Specificity using Femtosecond Resonant Inelastic X-ray Scattering at the Nitrogen K-Edge
}

Raphael M. Jay, ${ }^{1,+}$ Sebastian Eckert, ${ }^{1,2}$ Benjamin E. Van Kuiken, ${ }^{3,{ }^{*}}$ Miguel Ochmann, ${ }^{4}$ Markus Hantschmann, ${ }^{1,2}$ Amy A. Cordones, ${ }^{5, \%}$ Hana Cho, ${ }^{5,6, \S}$ Kiryong Hong, ${ }^{6, \$}$ Rory Ma, ${ }^{4,6, \&}$ Jae Hyuk Lee, ${ }^{5, \&}$ Georgi L. Dakovski, ${ }^{7}$ Joshua J. Turner, ${ }^{7,8}$ Michael P. Minitti, ${ }^{7}$ Wilson Quevedo, ${ }^{2}$ Annette Pietzsch, ${ }^{2}$ Martin Beye, ${ }^{2, \|}$ Tae Kyu Kim, ${ }^{9}$ Robert W. Schoenlein, ${ }^{5, \Delta}$ Philippe Wernet, ${ }^{10}$ Alexander Föhlisch ${ }^{1,2}$ and Nils Huse ${ }^{4, *}$

${ }^{1}$ Institut für Physik und Astronomie, Universität Potsdam, 14476 Potsdam, Germany ${ }^{2}$ Institute for Methods and Instrumentation for Synchrotron Radiation Research, HelmholtzZentrum Berlin für Materialien und Energie, 12489 Berlin, Germany

${ }^{3}$ European XFEL, 22869 Schenefeld, Germany

${ }^{4}$ Department of Physics, University of Hamburg and Center for Free-Electron Laser Science, 22761 Hamburg, Germany

${ }^{5}$ Ultrafast X-ray Science Lab, Chemical Sciences Division, Lawrence Berkeley National Laboratory, Berkeley, California 94720, United States

${ }^{6}$ Department of Chemistry and Chemistry Institute of Functional Materials, Pusan National University, Busan 46241, South Korea

${ }^{7}$ Linac Coherent Light Source, SLAC National Accelerator Laboratory, Menlo Park, CA 94025, USA

${ }^{8}$ Stanford Institute for Materials and Energy Sciences, Stanford University, Stanford, CA 94305, USA

${ }^{9}$ Department of Chemistry, Yonsei University, Seoul 03722, Republic of Korea

${ }^{10}$ Department of Physics and Astronomy, Uppsala University, 75120 Uppsala, Sweden

\section{Corresponding Authors}

*Email: benjamin.van.kuiken@xfel.eu

*Email: nils.huse@uni-hamburg.de

\section{Present Addresses}

${ }^{\dagger}$ Department of Physics and Astronomy, Uppsala University, 75120 Uppsala, Sweden

\%Stanford PULSE Institute, SLAC National Accelerator Laboratory, Menlo Park, California 94025, United States

$\S$ Inorganic Metrology Group, Division of Chemical and Biological Metrology, Korea Research Institute of Standards and Science, Daejeon, 34113, Republic of Korea 
\$Gas Metrology Group, Division of Chemical and Biological Metrology, Korea Research Institute of Standards and Science, Daejeon 34113, Republic of Korea

\&Pohang Accelerator Laboratory, Pohang, Gyeongbuk 37673, Republic of Korea

"Deutsches Elektronen-Synchrotron DESY, 22607 Hamburg

$\triangle$ Linac Coherent Light Source, SLAC National Accelerator Laboratory, Menlo Park, California 94025, United States

\section{Chemicals and materials}

Tris(2,2'-bipyridine)dichlororuthenium(II) hexahydrate ( $\mathrm{Ru}(\mathrm{bpy})_{3} \mathrm{Cl}_{2} \cdot 6 \mathrm{H}_{2} \mathrm{O}, 98.0 \%$ min titr. (Zn(II), EDTA)) was obtained from Chemos $\mathrm{GmbH}$ and recrystallized from isopropanol (Sigma Aldrich US, ACS reagent, $\geq 99.5 \%$ ). Water was purified by a Millipore Milli-Q purification system (Impedance $18.2 \mathrm{M} \Omega$ ). Prior to the measurement, the $50 \mathrm{mM}$ sample solutions were degassed using a sonication bath and filtered through Merck Steritop filters $(0.22 \mu \mathrm{m}$ pore size). Samples were typically replaced after 4 to 6 hours and checked for photodamage by UV/VIS absorption spectroscopy.

\section{Experimental details}

A liquid jet experimental station installed at the soft X-ray materials science (SXR) beamline of the Linac Coherent Light Source (LCLS) was used for femtosecond RIXS experiments as already described elsewhere ${ }^{1,2}$. Briefly, the sample solution was flowed through a $22 \mu \mathrm{m}$ wide cylindrical nozzle at $27 \mathrm{~m} / \mathrm{s}$ into the differentially pumped vacuum chamber. Circularly polarized laser pulses (480 nm wavelength, 50 fs FWHM duration, $10 \mu$ energy) where focused to $100 \mu \mathrm{m}$ at the sample position. Nearly collinear monochromatized X-ray pulses resonant to the $\mathrm{N}$ K-edge $(0.2 \mathrm{eV}$ FWHM bandwidth, $70 \mathrm{fs}$ FWHM duration, horizontally polarized, $120 \mathrm{~Hz}$ ) from the LCLS where focused to $30 \mu \mathrm{m}$, overlapping with the laser focus. The RIXS data were collected by a grating spectrometer based on Rowland-geometry at a $90^{\circ}$ detection angle within the plan of the polarization of the incident X-rays. At the N K-edge, this provided an energy resolution of $0.5 \mathrm{eV}$.

\section{Computational details}

Density functional theory (DFT) at the B3LYP level ${ }^{3,4}$ was used to calculate the equilibrium structure of $\left[\mathrm{Ru}(\mathrm{bpy})_{3}\right]^{2+}$ in the ground state as well as the ${ }^{3} \mathrm{MLCT}$ state using the ORCA quantum chemistry package ${ }^{5}$. For the excited state, DFT as a ground state theory is applicable because it is the energetically lowest state of triplet spin multiplicity. Throughout all calculations, the def2-TZVP basis set ${ }^{6}$ was employed including the standard Stuttgart-Dresden ECP for $\mathrm{Ru}^{7}$. The RIJCOSX approximation ${ }^{8}$ with the def2-TZV/J auxiliary basis ${ }^{9}$ set was used to speed up the calculations. The surrounding water solvent was modelled by the CPCM 
approach ${ }^{10}$. X-ray absorption transitions of the singlet ground state and the ${ }^{3} \mathrm{MLCT}$ state were modelled using time-dependent density functional theory (TD-DFT). The Pipek-Mezey orbital localization scheme ${ }^{11}$ is used to guarantee a localized core hole. The calculated transitions are convolved with a Lorentzian broadening of $0.13 \mathrm{eV}$ to account for the lifetime of the $\mathrm{N}$ 1s core-hole ${ }^{12}$. For best agreement with the main resonance of the ground state spectrum, an additional Gaussian broadening of $0.6 \mathrm{eV}$ is applied and all spectra are shifted by $12.5 \mathrm{eV}$.

RIXS planes of the ground state and ${ }^{3} \mathrm{MCLT}$ state have been calculated from a one-electron picture using the DFT calculations as input. Excited states were taken to be singly-excited slater determinants. The core-excited states are constructed by the promotion of a $\mathrm{N} 1 \mathrm{~s}$ electron to the valence space. The valence excitations span the entire occupied space which is necessary to calculate the RIXS spectra up to $\sim 25 \mathrm{eV}$ in energy transfer. In this approximation the Kohn-Sham orbital energy differences give the excitation energies. The transition dipole moments between these states reduce to a single transition dipole integral between two orbitals. The RIXS plane was constructed according to the Kramers-Heisenberg equation. The spectra are Gaussian broadened by $0.8 \mathrm{eV}$ along the energy transfer axis and shifted to match the inelastic part of the experimental spectrum. These RIXS planes were calculated using the X-ray emission spectroscopy module of Orca following the protocol given in Ref. 13.

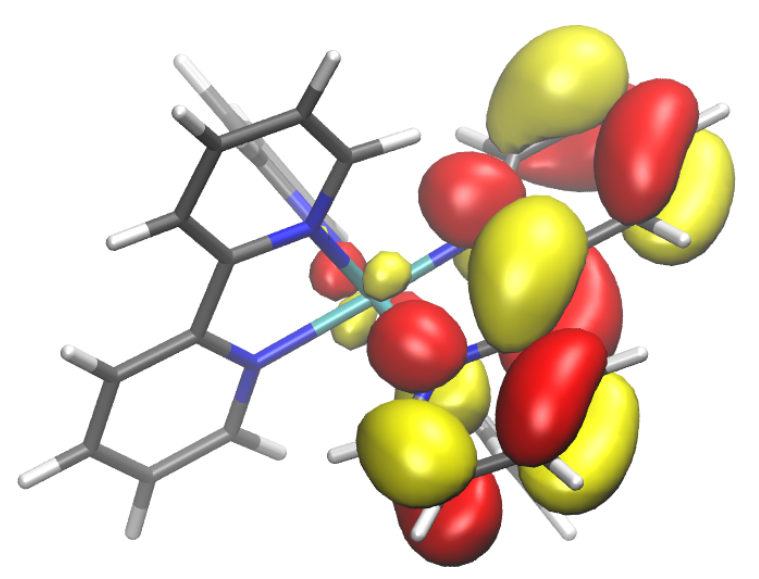

Fig. S1 Visualization of the highest occupied molecular orbital of the ${ }^{3} \mathrm{MLCT}$ state corresponding the orbital containing the transferred electron clearly localized on a single bpy group. 

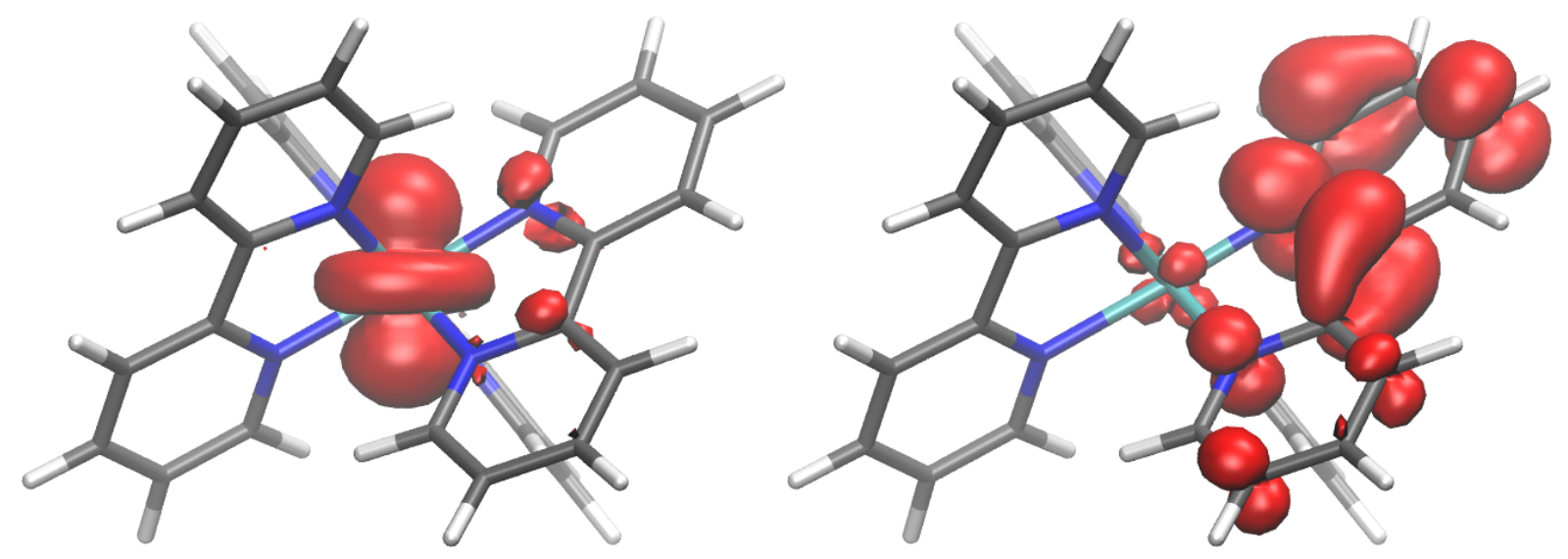

Fig. S2 Difference densities for the N1s $\rightarrow$ Ru $t_{2 \mathrm{~g}}$ (left) as well as the N1s $\rightarrow$ bpy $\pi^{*}$ (right) coreexcitation of the reduced bpy.
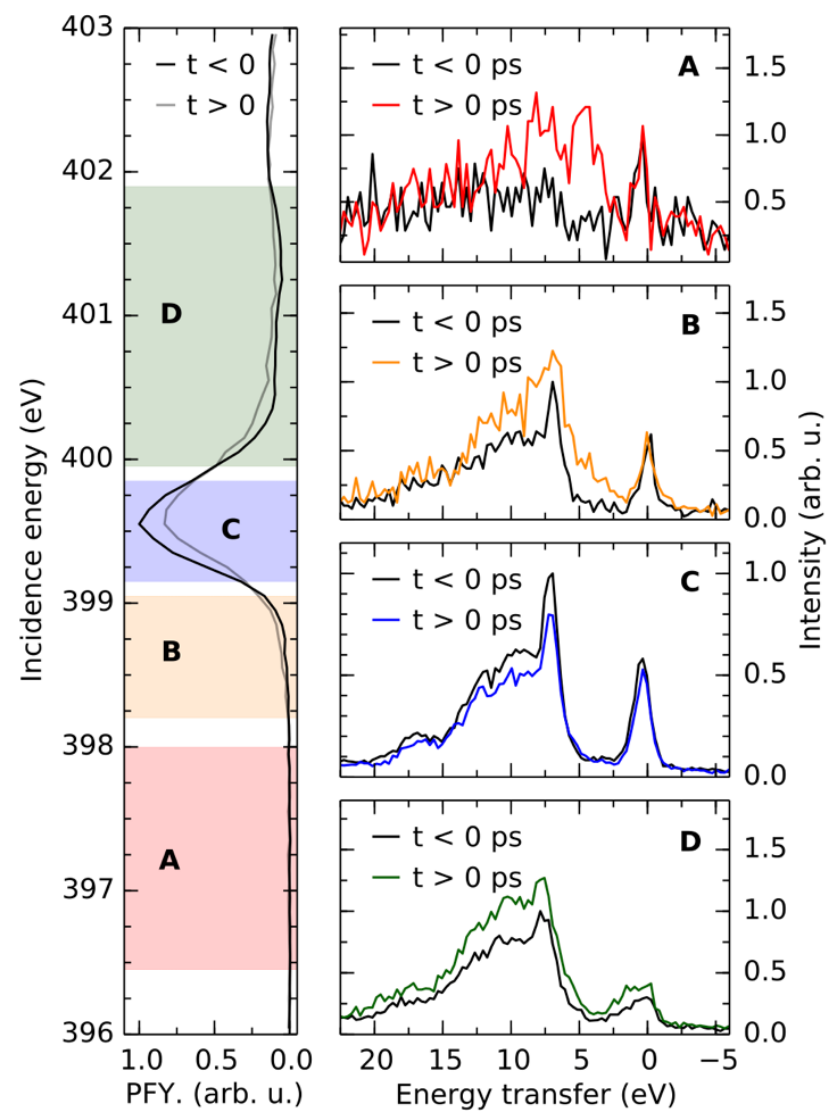

Fig. S3 RIXS data across the full measured incidence energy range including additional pre (B) and post edge regions (D) not discussed in the main text. 


\section{References}

(1) Schlotter, W. F.; Turner, J. J.; Rowen, M.; Heimann, P.; Holmes, M.; Krupin, O.; Messerschmidt, M.; Moeller, S.; Krzywinski, J.; Soufli, R. et al. THE SOFT X-RAY INSTRUMENT FOR MATERIALS STUDIES AT THE LINAC COHERENT LIGHT SOURCE XRAY FREE-ELECTRON LASER. Rev. Sci. Instrum. 2012, 83, 043107.

(2) Kunnus, K.; Rajkovic, I.; Schreck, S.; Quevedo, W.; Eckert, S.; Beye, M.; Suljoti, E.; Weniger, C.; Kalus, C.; Grübel, S. et al. A SETUP FOR RESONANT INELASTIC SOFT X-RAY SCATTERING ON LIQUIDS AT FREE ELECTRON LASER LIGHT SOURCES. Rev. Sci. Instrum. 2012, 83 (12), 123109.

(3) Becke, A. D. DENSITY-FUNCTIONAL EXCHANGE-ENERGY APPROXIMATION WITH CORRECT ASYMPTOTIC BEHAVIOR. Phys. Rev. A 1988, 38, 3098-3100.

(4) Becke, A. D. A NEW MIXING OF HARTREE-FOCK AND LOCAL DENSITY-FUNCTIONAL THEORIES. J. Chem. Phys. 1993, 98, 1372-1377.

(5) Neese, F. THE ORCA PROGRAM SYSTEM. Wiley Interdiscip. Rev. Comput. Mol. Sci. 2012, 2, 73-78.

(6) Weigend, F.; Ahlrichs, R. BALANCED BASIS SETS OF SPLIT VALENCE, TRIPLE ZETA VALENCE AND QUADRUPLE ZETA VALENCE QUALITY FOR H TO RN: DESIGN AND ASSESSMENT OF ACCURACY. Phys. Chem. Chem. Phys. 2005, 7, 3297-3305.

(7) Andrae, D.; Häußermann, U.; Dolg, M.; Stoll, H.; Preuß, H. ENERGY-ADJUSTABLE INITIO PSEUDOPOTENTIALS FOR THE SECOND AND THIRD ROW TRANSITION ELEMENTS. Theor. Chim. Acta 1990, 77, 123-141.

(8) Neese, F.; Wennmohs, F.; Hansen, A.; Becker, U. EFFICIENT, APPROXIMATE AND PARALLEL HARTREE-FOCK AND HYBRID DFT CALCULATIONS. A "CHAIN-OF-SPHERES" ALGORITHM FOR THE HARTREE-FOCK EXCHANGE. Chem. Phys. 2009, 356, 98-109.

(9) Weigend, F. ACCURATE COULOMB-FITTING BASIS SETS FOR H TO RN. Phys. Chem. Chem. Phys. 2006, 8, 1057-1065.

(10) Barone, V.; Cossi, M. QUANTUM CALCULATION OF MOLECULAR ENERGIES AND ENERGY GRADIENTS IN SOLUTION BY A CONDUCTOR SOLVENT MODEL. J. Phys. Chem. A 1998, 102, 1995-2001.

(11) Pipek, J.; Mezey, P. G. A FAST INTRINSIC LOCALIZATION PROCEDURE APPLICABLE FOR AB INITIO AND SEMIEMPIRICAL LINEAR COMBINATION OF ATOMIC ORBITAL WAVE FUNCTIONS. J. Chem. Phys. 1989, 90, 4916-4926.

(12) Campbell, J. L.; Papp, T. WIDTHS OF THE ATOMIC K-N7 LEVELS. At. Data Nucl. Data Tables 2001, 77, 1-56.

(13) Hall, E. R.; Pollock, C. J.; Bendix, J.; Collins, T. J.; Glatzel, P.; DeBeer, S. VALENCE-TOCORE DETECTED X-RAY ABSORPTION SPECTROSCOPY: TARGETING LIGAND SELECTIVITY. J. Am. Chem. Soc. 2014, 136, 10076-10084. 